\title{
GEOMETRY AND NONLINEAR ANALYSIS IN BANACH SPACES
}

\author{
KondagunTA SUNDARESAN
}

It is proved that if a Banach space admits a nontrivial uniformly continuously differentiable function with bounded support then it is superreflexive. Several applications to approximation theory and existence of solutions to differential equations are discussed.

A Banach space $E$ is said to be $U^{1}$-mooth if there exists a uniformly continuously differentiable real-valued function on $E$ with bounded support. It is of considerable importance to know whether a given Banach space $E$ is $U^{1}$-smooth since certain real-valued functions on $E$ cannot be approximated by smoother functions if $E$ fails to be $U^{1}$-smooth. Results of this type are of considerable interest in global analysis on infinite dimensional manifolds, see Eells [6], and Lang [11]. Motivated by these considerations and the connections of $U^{1}$-smoothness with Banach spaces with norms of class $C^{1}$-away from 0 , with Lipschitzian derivatives, Wells [16], Moulis [12], Heble [8], and Aron [1], among others, discussed this problem and related concepts. In [16], it is shown that the Banach space $C_{0}$ fails to be $U^{1}$-smooth, while in [1] it is proved that the Banach spaces $C(K), K$ an infinite compact Housdorff space are not $U^{1}$-smooth. The primary purpose of this paper is to completely characterize Banach spaces which are $U^{1}$-smooth in terms of the geometry of the space. The main result stated in the Theorem 3 here, implies that the class of Banach spaces which are not $U^{1}$-smooth is very extensive modulo isomorphism, includes a class of reflexive spaces, and the results in [1] and [16] are deduced as corollaries of the main theorem.

The plan of the paper is as follows. In $\S 1$ few known results and definitions relevant to the discussion here are recalled. In $\S 2$ the main results are established. Applications to approximation theory, differential equations on a Banach space, and related results are discussed in $\S 3$.

1. In this paper, unless otherwise specified, the same symbol || $\|$ is used for the norms of various Banach spaces that enter the discussion as this does not entail any confussion. If $E$ is a Banach space, the open ball, center 0 , and radius $r$ is denoted by $U_{r}(0)$. The region $\{x \mid \lambda<\|x\|<\mu\}$ in $E$ is denoted by $R(\lambda, \mu)$, for $0<\lambda<\mu$. If $U$ is any open set, the boundary of $U$ is denoted by $\partial U$. The 
support of a vector-valued function $f$ is the set $\{x \mid f(x) \neq 0\}$. Thus in this paper if $f$ is a continuous function defined on a topological space $X$ into a Banach spaces $E$, then its support is an open subset of $X$. If $E, F$ are Banach space, then the Banach space of continuous linear operators on $E$ into $F$ with the supremum norm, is denoted by $L(E, F)$.

If $E, F$ are Banach spaces $E$ is said to be finitely represented in $F$, in symbols $E \ll F$, if for each finite dimensional subspace $X$ of $E$, and positive number $\varepsilon$, there is a subspace $Y$ of $F$, depending on $X$ and $\varepsilon$, such that there is an isomorphism $T$ on $X$ onto $Y$ with $\|T\|\left\|T^{-1}\right\| \leqq 1+\varepsilon$. A Banach space $F$ is said to be superreflexive if $E \ll F$ implies $E$ is reflexive. For fundamental work on superreflexive spaces see James [10], and for a comprehensive account of these spaces the interested reader is referred to the recent monograph of Van Dulst [15] on the subject.

An useful concept in the theory of finite representation is the concept of an ultrapower of a normed linear space. Let $S$ be an infinite set and $\Gamma$ be a nontrivial (free) ultrafilter on $S$. If $f$ is a bounded real-valued function on $S$ let $\lim _{\Gamma} f(s)=\sup [\lambda \mid\{t \in S$, $f(t)>\lambda\} \in \Gamma]$. Now if $(E,\|\quad\|)$ is a normed linear space, and $f$ is a bounded $E$-valued function on $S$, let $|f|=\lim _{\Gamma}\|f(s)\|$. It is verified that | | is a seminorm on the vector space $V$ of bounded $E$-valued functions on $S$, and the quotient space of $V$ modulo the kernel of I | equipped with the quotient norm is known as the ultrapower of $E$ associated with the pair $(S, \Gamma)$, and is denoted here by $E(S, \Gamma)$. It is known that if $E$ is a Banach space, then $E(S, \Gamma)$ is a Banach space, and $E \ll F$ if and only if $E$ is isometric with a subspace of an ultrapower $F(S, \Gamma)$ of $F$. For an account of ultrapowers, see Stern [14]. $x \in E$

A Banach space $(E,\|\|)$ is said to be smooth if for all $x \neq 0$,

$$
\lim _{t \rightarrow 0} \frac{\|x+t y\|-\|x\|}{t}=G_{x}(y)
$$

exists for all $y \in E$. If the limit in (1) exists at a $x \neq 0$, for all $y \in E$, then it is known that $G_{x} \in E^{*}$, the dual of $E$, and $\left\|G_{x}\right\|=1$. A smooth Banach space $E$ is said to be uniformly smooth if the limit in (1) is attained uniformly for all $x, y,\|x\|=1=\|y\|$. It follows from the homogenity of the norm that a Banach space $E$ is uniformly smooth if and only if the norm is uniformly continuously differentiable on regions $R(\lambda, \mu)$, in particular on bounded sets at a positive distance from the origin. The following theorem of James and Enflo is useful in the discussion to follow. 
TheOREM 1. [James and Enflo]. A Banach space $E$ is superreflexive if and only if $E$ is isomorphic with an uniformly smooth Banach space.

For a formal proof, see [15]. See also in this connection [10], and Enflo [7].

Before concluding this section, the interested reader is referred to Dieudonné [5], Bourbaki [4] and [11], for various results in differential calculus in Banach spaces of which free use is made here.

2. In this section, the main results of the investigation are presented. The main result stated in Theorem 4 here completely characterizes the class of Banach spaces which are $U^{1}$-smooth in terms of the geometry of the space. For convenience the term "uniformly continuously differentiable" is often abbreviated as U.C.D. A few elementary lemmas essential in the subsequent discussion are presented to start with.

Lemma 1. Let $E$ be a Banach space and $f: E \rightarrow R$ be an uniformly continuously differentiable function, and let $D f$ be the differential of $f$. Then

(a) if $U$ is a bounded subset of $E$, then the restriction $f \mid U$ is Lipschitzian i.e., there is a positive number $M$ such that for $x, y \in U$, $|f(x)-f(y)| \leqq M\|x-y\|$,

(b) if the support of $f$ is bounded, then $f$ is globally Lipschitzian, in particular $f$ is uniformly continuous.

Proof. (a) Let $U_{r}(0)$ be an open ball such that $U_{r}(0) \supset U$. Since $f$ is U.C.D. and $U_{r}(0)$ is bounded, $\sup _{x \in U_{r}(0)}\|D f(x)\|<\infty$. Thus if $M$ is the preceeding supremum the mean value theorem implies that $|f(x)-f(y)| \leqq M\|x-y\|$ for $x, y \in U_{r}(0)$.

(b) is a consequence of (a).

Lemma 2. If $E$ is an $U^{1}$-smooth Banach space and $\lambda$ is a positive real number, then there is an uniformly continuously differentiable real-valued function $f$ on $E$ with $f(0)=1$, and $f(x)=0$ if $\|x\| \geqq \lambda$.

Proof. Since $E$ is $U^{1}$-smooth there is an U.C.D. real-valued function $g$ such that $g \neq 0$, and the support of $g$, say $U$, is a bounded subset of $E$. Let $a \in U$, and $\alpha$ be a positive number such that $\alpha(U-a) \subset U_{\lambda}(0)$. Define $f(x)=(1 / g(a)) g(x / \alpha+a)$. It is verified that the support of $f \subset U_{\lambda}(0)$, and that $f$ is an U.C.D. real-valued 
function with $f(0)=1$.

LEMMA 3. If $f$ and $g$ are two uniformly continuously differentiable real-valued functions on a Banach space $E$, and the support of $f$ or $g$ is bounded, then the product function $f g$ is an uniformly continuously differentiable function with bounded support.

Proof. For definiteness let the support of $f$, say $U$, be bounded. Let $\Delta$ be any positive number and $V=\{x \mid d(x, \bar{U})<\Delta\}$ where $\bar{U}$ is the closure of $U$, and $d(x, \bar{U})$ is the distance of $x$ from $\bar{U}$. Then $V$ is a bounded open set and $\bar{U} \subset V$. From Lemma 1 it sollows that there is a constant $M>0$ such that

(*) $\quad \operatorname{Max}\left\{\sup _{x \in E^{\prime}}|f(x)|, \sup _{x \in V}|g(x)|, \sup _{x \in E}\|D f(x)\|, \sup _{x \in V}\|D g(x)\|\right\} \leqq M$.

Now if $\varepsilon>0$, since $D f, D g$, and the restrictions $f|V, g| V$ are uniformly continuous, there is a $\delta>0$ such that $0<\delta<1 / 2 \Delta$, and if $\|x-y\|<\delta$ then $\|D f(x)-D f(y)\|<\varepsilon, \quad\|D g(x)-D g(y)\|<\varepsilon$ for $x, y \in E$, and $|f(x)-f(y)|<\varepsilon,|g(x)-g(y)|<\varepsilon$ if $x, y \in V$. Now noting that

$$
\begin{aligned}
\|D(f g)(x)-D(f g)(y)\| \leqq & \|D f(x)\||g(x)-g(y)|+|f(y)|\|D g(x)-D g(y)\| \\
& +\|D g(x)\||f(x)-f(y)|+|g(y)|\|D f(x)-D f(y)\|,
\end{aligned}
$$

$D(f g)(x)=0$ for $x \notin \bar{U},\|x-y\| \geqq \Delta>\delta$ if $x \in \bar{U}$ and $y \notin V$, and applying $\left(^{*}\right)$ it is verified from the choice of $\delta$, that if $\|x-y\|<\delta$ then $\|D(f g)(x)-D(f g)(y)\| \leqq 4 M \varepsilon$. Hence it follows that $f g$ is an U.C.D. function with bounded support.

Lemma 4. If $E, F, G$ are three Banach spaces, $f: E \rightarrow F$, and $g: F \rightarrow G$ are two uniformly continuously differentiable functions such that the derivatives $D f, D g$ are bounded mappings on $E \rightarrow$ $L(E, F)$, and on $F \rightarrow L(F, G)$ respectively then their composite $g \circ f$ : $E \rightarrow G$ is uniformly continuously differentiable.

Proof. The lemma follows by noting that

$$
\begin{aligned}
\|D(g \circ f)(x)-D(g \circ f)(y)\|_{3} \leqq & \|D g(f(x))\|_{2}\|D f(x)-D f(y)\| \\
& +\|D f(y)\|_{1}\|D g(f(x))-D g(f(y))\|_{2}
\end{aligned}
$$

where \|\|$_{i}, i=1,2,3$ are respectively the norms in the spaces $L(E, F), L(F, G)$, and $L(E, G)$, and the fact that $f$ is uniformly continuous since $D f$ is bounded.

LEMma 5. If $E$ is a uniformly convex and uniformly smooth 
Banach space, then the restrictions of the uniformly continuous differentiable functions on $E$ to any closed ball $\bar{U}_{r}(0)$ is dense in the space of uniformly continuously differentiable functions on $\bar{U}_{r}(0)$ with the uniform topology.

For a proof of this lemma, see Nemirovskii and Seminov [13].

REMARK 1. Before passing to the next lemma it is noted that if $E$ is uniformly smooth space, so that the norm of $E$ is uniformly continuously differentiable on regions $R(\lambda, \mu)$, it follows by composing the norm of $E$ with suitable $C^{1}$-function on $R \rightarrow R$ and using Lemma 4 that $E$ is $U^{1}$-smooth. Now applying Lemmas 2 and 4 , and using the fact that the norm of $E$ is U.C.D. on $R(\lambda, \mu)$, it is verified that if $E$ is uniformly smooth space, and $r, \varepsilon$ are two positive numbers, then there is an U.C.D. function $f: E \rightarrow R$ such that, $0 \leqq f \leqq 1$, $f \equiv 1$ on $U_{r}(0)$, and $f$ vanishes outside $\overline{U_{r+\varepsilon}(0)}$.

The next lemma is crucial in proving the Theorem 6 in this paper. The lemma follows from the preceeding remark, and Lemma 5. A proof of the lemma is sketched. Before proceeding to the lemma it is noted that a super-reflexive Banach space could be equipped with an equivalent norm which is uniformly convex, and uniformly smooth, [7].

LEMMA 6. If $G$ is a nonempty open subset of a super-reflexive space $E$, then there is an U.C.D. function $f: E \rightarrow R, 0 \leqq f \leqq 1$, with support of $f=G$.

Proof. In view of what is noted in the preceeding paragraph in proving the lemma, it can be assumed that $E$ is as in the lemma 5 .

As a primary case let $G$ be an open bounded set, and $C=E \sim G$, and $g: E \rightarrow R$ be the function $g(x)=d(x, C)$, where $d(x, C)$ is the distance of $x$ from $C$. Let $r>0$ be such that $G \subset \bar{G} \subset U_{r / 2}(0)$. Consider the restriction of the uniformly continuous function $g$ to the ball $\overline{U_{r}(0)}$. By Lemma 5, there are U.C.D. functions $f_{n}$ on $E \rightarrow R$, for integers $n \geqq 1$, such that $\sup _{x \in U_{r}(0)}\left|f_{n}(x)-g(x)\right|<1 / n$. Now using Remark 1, there is an U.C.D. function $\phi: E \rightarrow R, \phi \equiv 1$ on $\overline{U_{r / 2}(0)}, \phi \equiv 0$ outside $U_{r}(0)$. Let $h_{n}=\phi \cdot f_{n}$. Let $\alpha_{n}$ be $C^{1}$-functions on $R \rightarrow R$, with support $\left.\alpha_{n}=\right] 1 / n, \infty$ [, such that $\alpha_{n}(t)=0$ if $t \leqq 1 / n$, $\alpha_{n}(t)=1$ if $t$ is in $\left[2 / n, \infty\left[\right.\right.$, and $0 \leqq \alpha_{n} \leqq 1$, for integers $n \geqq 1$. Let $g_{n}=\alpha_{n}\left(h_{n}\right)$. Now if $g=\sum_{n \geqq 1} 1 / 2^{n} g_{n}, g$ is an U.C.D. function on $E \rightarrow R$, with support of $g=G$, and $0 \leqq g \leqq 1$, completing a proof of Lemma 6 if $G$ is bounded.

Now if $G$ is an arbitrary open set then $G=\bigcup_{n \geqq 1} G_{n}$, where 
$G_{n}=U_{n}(0) \cap G, n \geqq 1$. Since each $G_{n}$ is a bounded open set, from what has been proved above, there is an U.C.D. function $0 \leqq f_{n} \leqq 1$, with support $f_{n}=G_{n}$. Let $f=\sum_{n \geqq 1} 1 / 2^{n} f_{n}$. Then $f$ has all the desired properties.

The next two results show that $U^{1}$ smoothness is finitely inherited.

THEOREM 2. If $E$ is $U^{1}$-smooth, then every ultrapower $E(S, \Gamma)$ of $E$ is $U^{1}$-smooth.

Proof. Let the norms of $E$, and $E(S, \Gamma)$ be respectively \|\| , and $\|\mid\|$. Since $E$ is $U^{1}$-smooth there is an U.C.D. function $f$, $f \neq 0$, on $E \rightarrow R$ with its support in the unit ball of $E$, see Lemma 2. Let $\tilde{x} \in E(S, \Gamma)$, and $\{x(s)\}_{s \in S}$ be a representative of $\widetilde{x}$. Since $f$ is a bounded function $\lim _{\Gamma} f(x(s))$ exists. Let $\{y(s)\}_{s \in S}$ be another representative of $\tilde{x}$. Now $f$ is uniformly continuous by Lemma 1 . Hence if $\varepsilon>0$ there is a $\delta>0$ such that $\|x-y\|<\delta$ implies $|f(x)-f(y)|<\varepsilon$. Further since $\{x(s)\}_{s \in S}, \quad\{y(s)\}_{s \in S}$ represent the same equivalence class $\tilde{x} \in E\{S, \Gamma\}$, there is a $J_{\hat{o}} \in \Gamma$ such that $\|x(s)-y(s)\|<\delta$ if $s \in J_{\delta}$. Hence $|f(x(s))-f(y(s))|<\varepsilon$ for all $s \in J_{\delta}$. Thus $\lim _{\Gamma} f(x(s))=\lim _{\Gamma} f(y(s))$. Hence if $f^{*}(\widetilde{x})=\lim _{\Gamma} f(x(s)), f^{*}$ is a real-valued function on $E(S, \Gamma)$. Since the support of $f$ is in the unit ball of $E, f^{*}(\widetilde{x}) \neq 0$ implies that there is a set $J \in \Gamma$ such that $\|x(s)\| \leqq 1$ for all $s \in J$. Thus $\|\widetilde{x}\| \leqq 1$. Hence the support of $f^{*}$ is in the unit ball of $E(S, \Gamma)$.

Since $D f: E \rightarrow E^{*}$ is an uriformly continuous mapping with bounded range, by proceeding as in the proceeding paragraph it is verified that if $\widetilde{x}, \widetilde{y} \in E(S, \Gamma)$, and $\{x(s)\}_{s \in S},\{y(s)\}_{s \in S}$ are representatives of $\widetilde{x}, \quad \widetilde{y}$ respectively, then $\lim _{\Gamma} D f(x(s))(y(s))$ is independent of the representatives $\{x(s)\},\{(y(s))\}$ of $\widetilde{x}$ and $\widetilde{y}$. For $\widetilde{x}, \widetilde{y} \in E(S, \Gamma)$ define $l_{\tilde{x}}(\widetilde{y})=\lim _{\Gamma} D f(x(s))(y(s))$. It is verified that $l_{\tilde{x}}$ is a continuous linear functional on $E(S, \Gamma)$, since $D f$ is bounded. Now if $\widetilde{h} \in E(S, \Gamma)$, and $\{h(s)\}_{s \in S} \in \tilde{h}$, then

$$
\begin{aligned}
f^{*}(\widetilde{x}+\widetilde{h}) & =\lim _{\Gamma} f(x(s)+h(s)) \\
& =\lim _{\Gamma}\left\{f(x(s))+D f(x(s))(f(s))+\theta_{x(s)}(h(s))\right\},
\end{aligned}
$$

where it is noted that, since $f$ is an U.C.D. function that given $\varepsilon>0$, there is a $\delta>0$ such that $\left|\theta_{x}(y)\right| \leqq \varepsilon\|y\|$ if $\|y\| \leqq \delta$ for all $x \in E$. Let now $\|\widetilde{h}\| \leqq \delta$. Then there is a set $J \in \Gamma$ such that for all $s \in J, \quad\left|\theta_{x(s)}(h(s))\right| \leqq \varepsilon\|h(s)\|$. Hence $\lim _{\Gamma}\left|\theta_{x(s)}(h(s))\right| \leqq \varepsilon|\|\tilde{h}\||$ if $\|\tilde{h}\| \mid \leqq \delta$ and $f^{*}$ is differentiable at $\widetilde{x}$ with $D f^{*}(\widetilde{x})=l_{\tilde{x}}$.

Since $D f$ is an uniformly continuous map on $E \rightarrow E^{*}$, it is verified that the map $D f^{*}: E(S, \Gamma) \rightarrow(E(S, \Gamma))^{*}$ is uniformly continu- 
ous once again working with suitable members of $\Gamma$ as has been done in the preceeding parts of the proof. Thus $E(S, \Gamma)$ is $U^{1}-$ smooth.

CoRollary 1. If $E$ is $U^{1}$-smooth, and $F \ll E$, then $F$ is $U^{1-}$ smooth.

Proof. The corollary follows from the preceeding theorem together with the fact that $F \ll E$ if and only if $F$ is isometric with a subspace of some ultrapower $E(S, \Gamma)$ of $E$.

REMARK 2. Since a superreflexive Banach space is isomorphic with an uniformly smooth Banach space, and $U^{1}$-smoothness is invariant under isomorphisms it follows that if a Banach space $E$ is superreflexive, then it is $U^{1}$-smooth.

TheORem 3. If $E$ is an $U^{1}$-smooth Banach space, then it is reflexive.

Proof. Let $0<\theta<1$. Lemma 2 assures that there is an U.C.D. real-valued function $f$ on $E$ such that $f(0)=1$, and $f(x)=0$ if $\|x\| \geqq \theta / 4$. Since $f$ is U.C.D. if $0<\varepsilon<1$, there is a positive integer $M$ such that if $h \in E,\|h\| \leqq 1 / M$, then

$$
|f(x+h)-f(x)-D f(x)(h)| \leqq \varepsilon\|h\| \text {. }
$$

If possible let $E$ be nonreflexive. Then by a theorem of James, see Theorem 7 in [9], it follows that there is a set $X$ containing the set $W$ of positive integers, and a subspace $L$ of the Banach space $B(X)$ of bounded real-valued functions on $X$ with the supremum norm, isometric with $E$, admitting a sequence $\left\{z_{n}\right\}_{n \geq 1}$, such that for $n \geqq 1$

$$
\begin{array}{ll}
z_{n}(i)=\theta, & 1 \leqq i \leqq n, \quad i \in W, \\
z_{n}(i)=0, & i>n, \quad i \in W,
\end{array}
$$

and

$$
\left|z_{n}(t)\right| \leqq 1 \text { for } t \in X \sim W .
$$

Let $x_{n, 0}=1 / 2 z_{n}, x_{0, n}=-1 / 4 z_{n}$ for $n \geqq 1$, and $x_{n, k}=3 / 4 z_{n}-1 / 4 z_{n+k}$ if $n \geqq 1, k \geqq 1$. Clearly $\left\|x_{n, k}\right\| \leqq 1$ for all the pairs of integers $(n, k)$ for which $x_{n, k}$ is defined. Consider the polynomial path $P \subset L$ defined by

$$
P=\bigcup_{i=0}^{2^{M}-1}\left[x_{2^{1}-i, i}, x_{2^{n}-i-1, i+1}\right]
$$


where $M$ is the positive integer chosen to satisfy the inequality (A) in the preceeding paragraph. Consider the derivative $D f(0)$ of $f$ at 0 . By our choice of $x_{n, k}, D f(0)\left(x_{2}, H, 0\right)=0$ if and only if $D f(0)\left(x_{0,2}{ }^{M}\right)=0$, and $D f(0)\left(x_{2} H, 0\right)$ is positive (negative) if and only if $D f(0)\left(x_{0,2}, H\right)$ is negative (positive). Since $P$ is connected there is a $\xi \in P$ such that $D f(0)(\xi)=0$. If

then

$$
\xi \in\left[x_{2} I_{-i_{0}, i_{0}}, x_{2^{H}-i_{0}-1, i_{0}+1}\right],
$$

$$
\begin{aligned}
& \xi(j)=\frac{1}{2} \theta \text { if } 1 \leqq j \leqq 2^{M}-i_{0}-1, \quad j \in W, \\
& \xi(j)=-\frac{1}{4} \theta, \quad \text { if } 2^{M}-i_{0}+1 \leqq j \leqq 2^{M}, \quad j \in W, \\
& \xi(j) \in\left[-\frac{1}{4} \theta, \frac{1}{2} \theta\right] \text { if } j=2^{M}-i_{0}, \quad j \in W,
\end{aligned}
$$

and

$$
\|\xi\| \leqq 1
$$

Thus if $\xi\left(j_{0}\right) 1 / 2 \theta$ for some $j_{0} \in W, 1 \leqq j_{0} \leqq 2^{M}$ (which is the case if $\left.\xi\left(j_{0}\right) \in\right]-\theta / 4, \theta / 2\left[\right.$ or $\left.\xi\left(j_{0}\right)=-\theta / 4\right)$, then $\xi(j)=-1 / 4 \theta$ for all $j \in W$, $j_{0}+1 \leqq j \leqq 2^{M}$. Now if $2^{H}-i_{0}-1 \geqq 2^{M-1}$, choose $\xi_{1}=\xi / M$, otherwise $\xi_{1}=-\xi / M$. The $\xi_{1}$ thus chosen has the properties, $\left\|\xi_{1}\right\| \leqq 1 / M$, $D f(0)\left(\xi_{1}\right)=0$, and $\xi_{1}(j) \geqq \theta / 4 M$ for at least $2^{Y-1}$ values of $j \in W$, $1 \leqq j \leqq 2^{M}$.

Next consider the derivative $D f\left(\xi_{1}\right)$. Then as before there is a $\xi^{\prime} \in P$ such that $D f\left(\xi_{1}\right)\left(\xi^{\prime}\right)=0$. From the properties of $\xi$ noted in the preceeding paragraph, since $\xi_{1}= \pm \xi / M$, the restriction of $\xi_{1}$ to the set $Q=\left\{j \mid 1 \leqq j \leqq 2^{M}\right\} \subset W$, has range either in the set $\{\theta / 2 M,-\theta / 4 M\}$ or $\{-\theta / 2 M, \theta / 4 M\}$ except possibly for one value of $j \in Q$. These observations imply either (i) $\left(\xi_{1}+\xi^{\prime} / M\right)(j) \geqq 2 \theta / 4 M$ or (ii) $\left(\xi_{1}-\xi^{\prime} / M\right)(j) \geqq$ $2 \theta / 4 M$ for at least $2^{M-2}$ integers $j \in Q$. Let $\xi_{2}=\xi^{\prime} / M$ or $-\xi^{\prime} / M$ according as (i) or (ii) is the case. Repeating this procedure inductively it follows that there is a sequence $\left\{\xi_{i}\right\}_{i=1}^{M}$ in $L$ such that $\left\|\xi_{i}\right\| \leqq 1 / M$, $D f\left(\sum_{i=1}^{k-1} \xi_{i}\right)\left(\xi_{k}\right)=0, \sum_{i=1}^{k} \xi_{i}(j) \geqq k \theta / 4 M$ for $1 \leqq k \leqq M$. From our choice of $f, M,\left\{\xi_{i}\right\}_{i=1}^{M}, \varepsilon$, together with the inequality $\left\|\sum_{i=1}^{k} \xi_{i}\right\| \geqq k \theta / 4 M$ it follows that

$$
\begin{aligned}
1+\left|f\left(\sum_{i=1}^{M} \xi_{i}\right)-f(0)\right| & \leqq \sum_{k=1}^{M}\left|f\left(\sum_{i=1}^{k} \xi_{i}\right)-f\left(\sum_{i=1}^{k-1} \xi_{i}\right)-D f\left(\sum_{i=1}^{k-1} \xi_{i}\right)\left(\xi_{k}\right)\right| \\
& \leqq \sum_{k=1}^{M} \varepsilon\left\|\xi_{i}\right\| \leqq \varepsilon<1,
\end{aligned}
$$

a contradiction, completing the proof of the theorem. 
The next theorem provides the characterization of $U^{1}$-smooth Banach spaces.

THEOREM 4. A Banach space $E$ is $U^{1}$-smooth if and only if $E$ is superreflexive.

Proof. From Corollary 1, and Theorem 3 it follows that if $E$ is $U^{1}$-smooth, and $F \ll E$, then $F$ is reflexive. Thus $E$ is superreflexive. The converse follows from the Remark 1.

Since the Banach spaces $C_{0}, C(K), K$ an infinite compact Hausdorff space are not superreflexive (not even reflexive) it follows that the theorems of Wells [15] and Aron [1] follow as corollaries from the preceeding characterization.

Corollary 2. If $E=C_{0}$, or $C(K)$ with $K$ as above, then $E$ is not $U^{1}$-smooth.

3. In this section several applications of the results in the $\S 2$ are discussed. Before proceeding to the applications of approximation theory and differential equations, a characteristic property of uniformly continuously differentiable functions, valid when the domain and the range are suitably chosen, is obtained.

THEOREM 5. If $E$ is a nonsuperreflexive Banach space, and $F$ is a superreflexive Banach space, and $f: E \rightarrow F$ is an uniformly continuously differentiable function then for every bounded open set $U \subset E, f(\partial U)$ is dense in $f(U)$. Further if $f_{1}, f_{2}$ are two uniformly continuously differentiable maps on $E \rightarrow F$, coinciding on $\partial U$ then $f_{1}=f_{2}$ on $U$.

Proof. Let $x \in U$. If possible let $f(x) \notin \overline{f(\partial U)}$, the closure of $f(\partial U)$. Then there is a ball $U_{s}$ with centre at $f(x)$ and an open set $G \supset \overline{f(\partial U)}$, such that $U_{\varepsilon} \cap G=\varnothing$. Since $F$ is $U^{1}$-smooth, Lemma 2 assures that there is an U.C.D. real-valued function $\psi$ on $F$ with support in $U_{\varepsilon}$, and $\psi(f(x))=1$. Let $g: E \rightarrow R$ be defined by $g(z)=$ $\psi(f(z))$ if $z \in U$, and $g=0$ on the set $E \sim U$. Since $\psi$ vanishes in a neighborhood of $\overline{f(\partial U)}$, it is clear that $g$ is a $C^{1}$-function. Now considering the halo $V=\{z \mid d(z, \bar{U})<\Delta\}$ for some $\Delta>0$, and the inequality

$$
\begin{aligned}
\|D g(y)-D g(z)\|_{1} \leqq & \|D \psi(f(y))\|_{2}\|D f(y)-D f(z)\|_{3} \\
& +\|D f(z)\|_{3}\|D \psi(f(y))-D \psi(f(z))\|_{2}
\end{aligned}
$$

where \|\|$_{i}, i=1,2,3$ are respectively the norms in the spaces $E^{*}$, $F^{*}$, and $L(E, F)$, and arguing as in the proof of Lemma 3 it follows 
that $g$ is an U.C.D. real-valued function on $E$ with its support in the bounded set $U$. Thus $E$ is $U^{1}$-smooth. Hence by Theorem 4, $E$ is superreflexive contradicting the hypothesis on $E$. Thus $f$ has the density as stated. The second part of the theorem is a direct consequence of the first part.

The following two propositions assert that certain smooth approximations are not possible. Similar results are known in the literature, see for example [1], however for very special cases, and the results in [1] follow as corollaries from the propositions here.

Proposition 2. Let $E, F$ be two Banach spaces and $E$ be nonsuperreflexive, and $F$ be superreflexive. If $p: E \rightarrow F$ is a function such that $p(x) \rightarrow 0$ as $\|x\| \rightarrow \infty$, then there is no nontrivial $C^{2}$-function $f$ with bounded second derivative on $E \rightarrow F$ such that $\|f(x)\|_{1} \leqq$ $\|p(x)\|_{1}$ where \|\| , and \|\|$_{1}$ are respectively the norms in the spaces $E$ and $F$.

Proof. Let $f: E \rightarrow F$ be a nontrivial $C^{2}$-function such that $\|f(x)\|_{1} \leqq\|p(x)\|_{1}$. Let $x_{0} \in E$ with $f\left(x_{0}\right) \neq 0$, and $R$ be a positive number such that if $\left\|x-x_{0}\right\| \geqq R$, then $\|p(x)\|_{1} \leqq 1 / 2\left\|f\left(x_{0}\right)\right\|_{1}$. From Theorem 5 it follows that if $U=\left\{x\|\| x-x_{0} \|<R\right\}$ then $f(\partial U)$ is dense in $f(U)$ but this contradicts the fact that $\|f(x)\|_{1} \leqq\|p(x)\|_{1} \leqq$ $1 / 2\left\|f\left(x_{0}\right)\right\|_{1}$ for $x \in \partial U$, completing the proof.

Proposition 3. Let $E, F$ be as in the preceeding proposition, and $p: E \rightarrow F$ be a bounded function with $p(x) \rightarrow 0$ as $\|x\| \rightarrow \infty$. If $f$ is a $C^{2}$-function on $E \rightarrow F$, and if $f$ is not a $C^{3}$-function there does not exist a $C^{3}$-function $g$ on $E$ into $F$ such that (a) $\|f(x)-g(x)\|_{1} \leqq$ $\|p(x)\|_{1}$, and (b) $\left\|D^{2} f(x)-D^{2} g(x)\right\|_{2} \leqq\|p(x)\|_{1}$ where, \|\| and \|\|$_{1}$ are the norms of $E$ and $F$, and \|\|$_{2}$ is the norm in the space $L(E, L(E, F))$.

Proof. If possible let there be a function $g: E \rightarrow F$ of class $C^{2}$ satisfying the inequalities (a) and (b). Since $f$ is not of class $C^{3}$, $f \neq g$. Thus it may be assumed that $\|f(0)-g(0)\|_{1}=a>0$. From (a) and (b) it follows that $(f-g)$ is a nontrivial differentiable function with a Lipschitzian derivative. Since $p(x) \rightarrow 0$ as $\|x\| \rightarrow \infty$, the inequality $\|f(x)-g(x)\|_{1} \leqq\|p(x)\|_{1}$ contradicts the preceeding proposition, completing the proof.

A well known theorem of Anderson and Kadec, see Bessaga and Pelczynski [2], asserts in part that all separable Banach spaces are homeomorphic. However the next corollary implies the nonexistence of U.C.D. homeomorphisms between certain Banach spaces, separable or not. 
CoROLlaRY 3. If $E, F$ are Banach spaces with $F$ superreflexive and if there is an uniformly continuously differentiable homeomorphism on $E$ into $F$, then $E$ is also superreflexive.

Proof. Let $f: E \rightarrow F$ be an U.C.D. homeomorphism. If possible let $E$ be nonsuperreflexive. Hence $E$ is not $U^{1}$-smooth. Consider the open balls $U_{r}=\{x|| \mid x \|<r\}$ in $E$. The hypothesis on $f$ implies $f\left(\partial U_{1}\right)$, and $f\left(\partial U_{2}\right)$ are disjoint. However from Theorem 5 it is inferred that $f(0) \in f\left(\partial U_{1}\right) \cap f\left(\partial U_{2}\right)$ since $f$ is an U.C.D. homeomorphism, a contradiction, completing the proof.

The preceeding results on smooth approximations have interesting applications in the context of differential equations on Banach spaces. In the next example the essential part in one such application is indicated.

ExAmple. Consider the problem of finding solutions $F$ for the equation $D^{2} F(x)=\alpha(\|x\|) Q(x)$ where $F$ is a real-valued function on a Banach space $E$, with $F(x) \rightarrow 0$ as $\|x\| \rightarrow \infty, \alpha$ is a nontrivial continuous function on $R \rightarrow R$ with its support in a compact set, $Q$ is a bounded continuous function on $E$ into the space of continuous symmetric bilinear forms on $E$, such that $\alpha(\|x\|) Q(x) \neq 0$ for at least one point $x \in E$. Let $E$ be a nonsuperreflexive Banach space. If possible let the equation admit a solution $F$. Since $\sup _{x \in E}\left\|D^{2} F(x)\right\|<$ $\infty$, and $|F(x)| \rightarrow 0$ as $\|x\| \rightarrow \infty$, it follows from Proposition 2, that $F$ must be 0 identically. Since $D^{2} F(x) \neq 0$ for at least one $x$, the equation does not admit a solution vanishing at infinity, if $E$ is not a superreflexive space.

The paper is concluded with the following theorem concerning partitions of unity and superreflexive spaces.

THEOREM 6. If $E$ is a superreflexive Banach space, and $\mathscr{G} i s$ an open covering of $E$, there exists a locally finite family of U.C.D. functions which is a partition of unity subordinated to $\mathscr{G}$.

The proof of Theorem 6 is completed by using Lemma 6 . Once Lemma 6 is available, by using standard techniques of point set topology to establish locally finite partitions of unity, the proof is completed.

The converse of Theorem 6 follows at once from Theorem 4 here.

\section{REFERENCES}

1. R. M. Aron, Approximation of differentiable functions, Infinite Dimensional Holomorphy, Math. Studies 12, North Holland Publishing Co., Amsterdam, 1977, 1-12. 
2. C. Bessaga and A. Pelczynski, Selected topics in infinite dimensional topology, Polish Scientific Publications, Warszawa, 1975.

3. R. Bonic and J. Frampton, Smooth functions on Banach manifolds, J. Math. Mech., 15 (1966), 877-898.

4. N. Bourbaki, Variétiés Différentiellés et Analytiques, Fascieule de Résultats, XXXIII and XXXIV, Herman, Paris, 1972.

5. J. Dieúdonné Foundations of Modern Analysis, Academic Press, New York, 1960.

6. J. Eells, Jr., A setting for global analysis, Bull. Amer. Math. Soc., 72 (1966), 751-807.

7. P. Enflo, Banach spaces which can be given an uniformly convex norm, Israel J. Math., 13 (1972), 281-288.

9. M. P. Heble, Lie theory and applications to entropy and approximation, Proceedings of the Conference on Lie Theory and Applications, Queens University, Kingston, Canada, (1977), 439-471.

9. R. C. James, Weak compactness and reflexivity, Israel J. Math., 2 (1964), 101-119. 10. - Some self dual properties of normed linear spaces, Symposium on Infinite Dimensional Topology, Ann. Math. Studies, 69 (1972), 159-175.

11. S. Lang, Differential Manifolds, Addison-Wesley, Reading, Mass., 1972.

12. N. Moulis, Approximation de fonctions differentiales sur certain espaces de Banach, Ann. Inst. Fourier, 21 (1971), 293-345.

13. A. M. Nemirovskii and S. M. Seminov, On polynomial approximation of functions in a Hilbert space, Sbornik U.S.S.R., 21 (1973), 255-277.

14. J. Stern, Some applications of model theory in Banach space theory, Annals of Math. Logic, 9 (1976), 49-121.

15. D. Van Dulst, Reflexive and Superreflexive Banach Spaces, Math. Centre Tracts 102, Mathematisch Centrum, Amsterdam, 1978.

16. J. Wells, Differentiable functions on $C_{0}$, Bull. Amer. Math. Soc., 75 (1969), 117-118. 17. D. Wulbert, Approximation of $C^{k}$-functions, Proc. Symposium on Approximation Theory, Austin, Texas, (1973), 217-239.

Received August 5, 1980 and in revised form July 31, 1981. This research was supported in part by a Cleveland State University Faculty Research Grant.

Cleveland State University

Cleveland, OH 44115 